\title{
Anti-NMDA receptor encephalitis: report of ten cases and comparison with viral encephalitis
}

\author{
M. S. Gable • S. Gavali • A. Radner • D. H. Tilley • \\ B. Lee $\cdot$ L. Dyner $\cdot$ A. Collins $\cdot$ A. Dengel $\cdot$ J. Dalmau • \\ C. A. Glaser
}

Received: 9 May 2009 / Accepted: 1 August 2009 / Published online: 29 August 2009

(C) The Author(s) 2009. This article is published with open access at Springerlink.com

\begin{abstract}
The California Encephalitis Project (CEP), established in 1998 to explore encephalitic etiologies, has identified patients with $N$-methyl-D-aspartate receptor (NMDAR) antibodies, the likely etiology of their encephalitis. This study compares the presentation of such patients to those with viral encephalitis, so that infectious disease clinicians may identify individuals with this treatable disorder. Patients were physician-referred, and standardized forms were used to gather demographic, clinical, and laboratory data. Features of anti-NMDAR+ patients were compared with the viral encephalitides of enteroviral (EV), rabies, and herpes
\end{abstract}

M. S. Gable

Department of Psychiatry, University of California San Francisco,

Fresno, CA, USA

S. Gavali $\cdot$ C. A. Glaser

Viral and Rickettsial Disease Laboratory,

Division of Communicable Disease Control,

Center of Infectious Disease,

California Department of Public Health,

Richmond, CA, USA

A. Radner

Salinas Valley Memorial Hospital,

Salinas, CA, USA

D. H. Tilley

Division of Infectious Disease, Naval Medical Center San Diego,

San Diego, CA, USA

B. Lee

Division of Infectious Disease,

Children's Hospital and Research Center Oakland,

Oakland, CA, USA

L. Dyner

Lucile Packard Children's Hospital,

Palo Alto, CA, USA simplex-1 (HSV-1) origins. Sixteen cases with confirmed viral etiologies were all negative on NMDAR antibody testing. Ten anti-NMDAR + patients were profiled with a median age of 18.5 years (range 11-31 years). None were Caucasian. They had a characteristic progression with prominent psychiatric symptoms, autonomic instability, significant neurologic abnormalities, and seizures. Two had a teratoma, and, of the remaining eight, four had serologic evidence of acute Mycoplasma infection. The clinical and imaging features of anti-NMDAR+ patients served to differentiate this autoimmune disorder from HSV-1, EV,
A. Dengel

Loma Linda University Medical Center, Loma Linda, CA, USA

\author{
J. Dalmau \\ Department of Neurology, Division of Neuro-oncology, \\ University of Pennsylvania, \\ Philadelphia, PA, USA
}

M. S. Gable $(\bowtie) \cdot$ C. A. Glaser $(\bowtie)$

Viral and Rickettsial Disease Laboratory, California Department of Health Services, 850 Marina Bay Parkway,

Richmond, CA 94804, USA

e-mail: ms2gable@yahoo.com

e-mail: Carol.glaser@cdph.ca.gov 
and rabies. Unlike classic paraneoplastic encephalitis, antiNMDAR encephalitis affects younger patients and is often treatable. The association of NMDAR antibodies in patients with possible Mycoplasma pneumoniae infection warrants further study.

\section{Introduction}

The causes of encephalitis are numerous, and most patients undergo extensive testing to identify infectious etiologies. However, other etiologies need to be considered. In the late 1960s, an association between limbic encephalitis and malignancy was made, and, since then, a number of antineuronal antibodies, such as the intracellular anti-Hu and anti-Ma, have been discovered and found to be associated with a specific array of cancers, as well as neuropsychiatric symptoms $[1,2]$. More recently, antibodies to neuronal extracellular membrane antigens have been recognized. One of these is the NR1 subunit of the $N$-methyl-D-aspartate receptor (NMDAR), which was first identified in 2007. Unlike its classic paraneoplastic counterparts, patients with this antibody may or may not have an associated neoplasm, and often respond to immunotherapy and tumor removal, if present [2].

The California Encephalitis Project (CEP) was initiated in 1998 to identify the etiologic agents and define the clinical and epidemiologic characteristics associated with encephalitis. Over 3,000 cases have been referred to the CEP, and many have been characterized into clinical profiles [3]. In 2007, the CEP was notified that one referred patient was diagnosed with an ovarian teratoma associated with NMDAR antibodies. This case, in combination with previous experience with cases of a similar phenotype and unclear etiology, as well as the increasing recognition of anti-NMDAR encephalitis worldwide, led us to a collaborative work with the University of Pennsylvania focused on the identification of similar cases within the context of CEP referrals $[2,4-7]$. The purpose herein is to describe patients within the CEP that tested positive for the NMDAR antibody and compare them with patients that had a confirmed viral etiology, so that infectious disease physicians can more readily identify those affected with this new, potentially treatable, immune-mediated disorder.

\section{Methods}

Patient selection/enrollment

Specimens are referred to the CEP by their treating physicians for diagnostic testing. Patients are required to be immunocompetent, at least 6 months of age, and must meet the CEP case definition of encephalitis, by which patients must be hospitalized with encephalopathy with at least one of the following: fever, seizure, focal neurologic findings, cerebrospinal fluid (CSF) pleocytosis, or electroencephalographic or neuroimaging findings consistent with encephalitis. A standardized case history form is submitted by the referring physician with patient information on exposures, travel, laboratory findings, and clinical and demographic characteristics. This study was approved by an ethical standards committee, and subjects signed a consent form prior to NMDAR antibody testing.

\section{CEP core testing}

CSF, respiratory specimens, and acute- and convalescentphase serum specimens are submitted and tested for 16 potential infectious agents of encephalitis, including herpesviruses, enteroviruses, arboviruses, respiratory viruses, and Mycoplasma pneumoniae [3].

Once the CEP became aware of the occurrence of antiNMDAR + encephalitis, collaboration with the University of Pennsylvania was established to facilitate the recognition and testing of anti-NMDAR+ cases within the CEP, as follows: retrospective testing of patients who had idiopathic encephalitis, along with dyskinesias or movement disorders; and prospective referral of cases based on similar syndromic features. Testing for NMDAR antibodies was performed as previously described [6]. Samples from a limited number of patients with confirmed viral etiologies including enteroviral (EV), rabies, and herpes simplex-1 (HSV-1), varicella zoster virus, and rabies were selected and tested for NMDAR antibodies.

\section{Data analysis}

Demographic, clinical, and laboratory data from antiNMDAR+ patients were compared with data from encephalitis patients with confirmed EV and HSV-1, since these are the most commonly identified viral etiologies within the CEP. Rabies virus cases were also included for comparison because this diagnosis was strongly suspected by referring physicians in several cases that were ultimately found to be NMDAR antibody positive. Categorical data were analyzed using the Chi-square test, and continuous data were subjected to the Kruskal-Wallis test. Statistical significance was defined as $P<0.05$.

\section{Results}

Characteristics of anti-NMDAR + cases

Of the 20 cases tested, ten anti-NMDAR + cases were identified; two patients were identified by retrospective 
testing and eight cases were identified prospectively. All ten patients were negative for viral studies, but four had a positive serum Mycoplasma IgM. The demographic, clinical, laboratory, and imaging findings of the ten cases are summarized in Table 1, while the frequencies are found in Table 2. The median age was 18.5 years (range 11-31 years), and none were of Caucasian descent, while there was a predilection for Asians/Pacific Islanders (50\%). Although $60 \%$ of patients presented with apparently benign symptoms, all had returned with more severe symptomatology within days. Nearly all patients demonstrated personality changes, and the majority (70\%) displayed extreme irritability. Psychiatric symptoms were common and manifested earlier in the course of the illness, often at presentation. Psychotic symptoms were noted in $68 \%$ of subjects, with all of these patients experiencing hallucinations, particularly of the auditory nature. Other schizophrenia-like symptoms, including flat affect and disorganized thinking, were reported in three patients. Two subjects presented with seizures, and an additional $60 \%$ developed seizures after hospitalization, often of the tonic-clonic type, but sometimes in combination with focal episodes. Mental status changes were nearly universal, and care in the intensive care unit (ICU) was required; patients had a median Glasgow Coma Scale (GCS) score of 8.

Choreoathetoid movements, ataxic gait, cranial nerve abnormalities, repetitive orofacial movements, and aphasia were each reported in around half of the patients. Subjects suffered from various combinations of neurologic findings, but $90 \%$ of patients experienced at least two or more abnormalities, while $60 \%$ demonstrated three or more abnormal neurologic findings. Sixty percent of those with orofacial dyskinesias had movements limited to the mouth alone, including lip smacking, tongue protrusion, and bruxism, each unassociated with a seizure focus on EEG when concurrent motor activity was present. None of the patients were believed to acquire these findings as a result of anti-psychotic medications, but, in one case, the medication did precede the development of symptoms. Choreoathetoid movements and dyskinesias were also highly variable from patient to patient, with no distinct pattern in terms of rate or rhythm (Table 1). Dysautonomia was nearly ubiquitous in anti-NMDAR+ patients, observed in $89 \%$ of cases. Tachycardia and bradycardia, blood pressure lability, hyperthermia, hypoventilation, and/or apnea were each reported in most patients with autonomic instability. Many patients experienced three or more types of instability, making these symptoms prominent clinical manifestations (Table 1).

Laboratory, EEG, and neuroimaging findings

The majority of anti-NMDAR+ patients $(89 \%)$ demonstrated a lymphocytic pleocytosis during the course of illness, with a median CSF white blood cell (WBC) count of $26 / \mu 1$ on presentation. The median CSF protein concentrations were elevated, but the CSF glucose was often within normal limits. EEG monitoring revealed diffuse slowing almost universally. Focal epileptic activity was uncommon (10\%) and paroxysmal discharges were not observed (Table 1).

Computed tomography $(\mathrm{CT})$ rarely revealed abnormalities. While magnetic resonance imaging (MRI) was normal on admission in all patients, changes were demonstrated in $40 \%$ of subjects on follow-up studies. However, the findings on MRI, for both fluid-attenuated inversion recovery (FLAIR) and T2-weighted images, were not consistent from patient to patient. Periventricular white matter demyelination and other nonspecific findings were observed in one case, and temporal lobe abnormalities were notable in others. Nonspecific abnormalities, or, often, no abnormality at all, appeared to be the standard for these patients.

Comparison of anti-NMDAR encephalitis with viral encephalitis

Samples from 16 cases with confirmed viral etiologies were all negative upon testing for anti-NMDAR antibodies. Comparison of anti-NMDAR + patients to those with viral etiologies of encephalitis revealed significant differences (Table 2). Viral cases were not disproportionately common among Asians or Pacific Islanders $(P<0.05$ for HSV-1, EV, and rabies-associated encephalitides). Psychosis was significantly less common in all viral groups with frequencies of 0 to $2 \%(P<0.0001)$, and the presence of hallucinations was also less likely $(P<0.05$ for all groups). Personality changes, though more frequent in HSV-1 and rabies virus patients, were seen significantly less often in encephalitic subjects with a viral etiology $(P<0.0001$ for all groups). Seizure activity was observed less often among virus-infected individuals, though not statistically less so among those with HSV-1 encephalitis $(P<0.001$ for EV and rabies cases).

Laboratory studies revealed that the CSF WBC median was higher in those with EV and HSV-1 (90 and 56 cells per $\mu$, respectively) than in those with anti-NMDAR+ encephalitis (22 cells per $\mu \mathrm{l})$. Rabies virus cases rarely demonstrated CSF pleocytosis, with a median count of 3.5 cells per $\mu \mathrm{l}(P<0.05)$. The median CSF protein concentrations were significantly higher in those with viral causes, with the exception of those with rabies $(65 \mathrm{mg} / \mathrm{dl}$ in HSV-1 and $54 \mathrm{mg} / \mathrm{dl}$ in EV vs. $28 \mathrm{mg} / \mathrm{dl}$ in anti-NMDAR+ patients; $P<0.05$ for both), but the CSF glucose concentrations did not differ. Temporal lobe changes on neuroimaging were more common in patients with HSV-1 (27 and 69\%, respectively; $P<0.05$ ).

Overall, viral causes, and especially EV encephalitis, appeared to cause fewer severe complications, including a lower rate of alteration in consciousness (36 vs. 50\%), ICU admission (33 vs. 80\%), and intubation (5 vs. 60\%). 


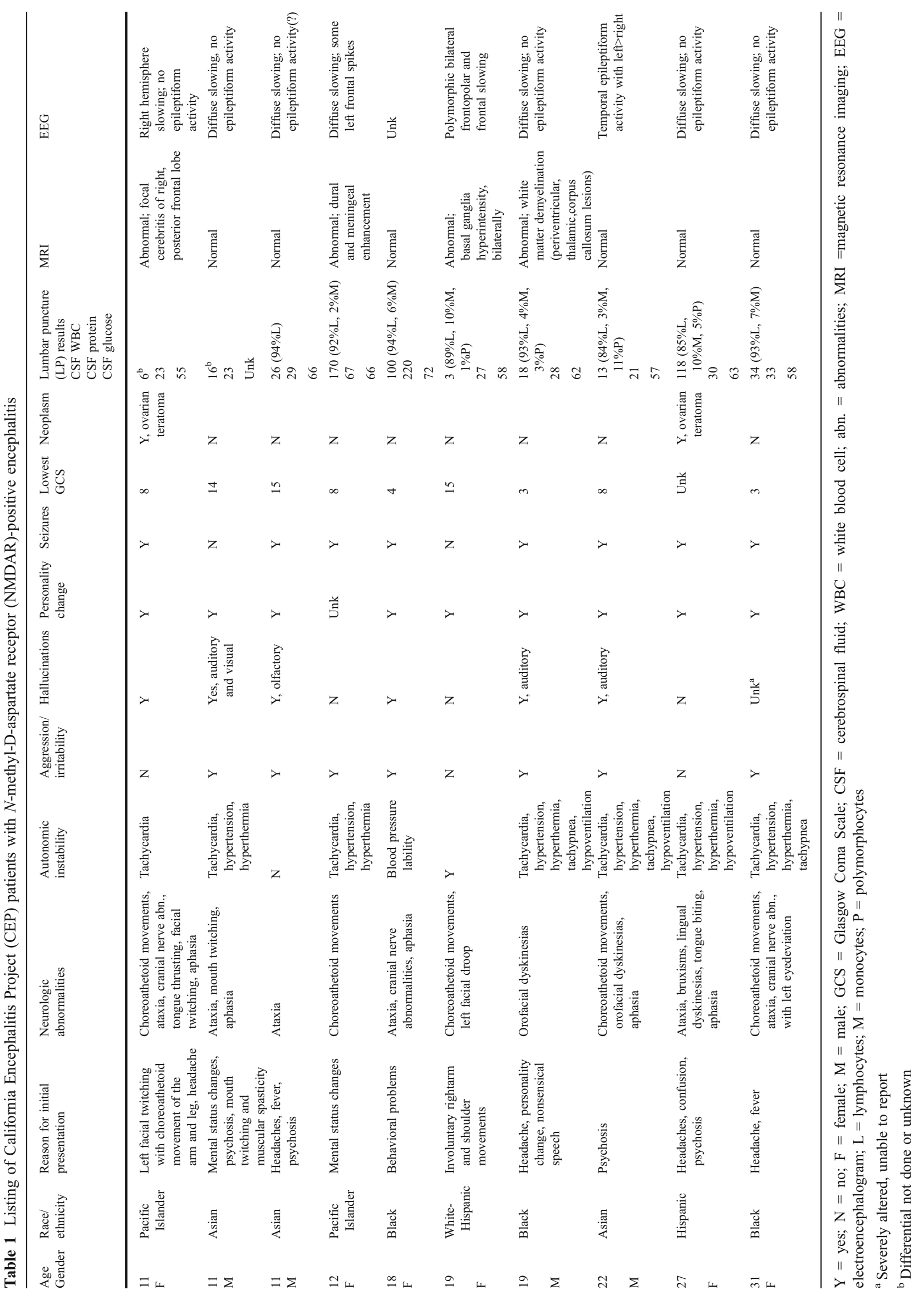


Table 2 CEP NMDAR cases vs. confirmed viral cases

\begin{tabular}{|c|c|c|c|c|}
\hline & $\operatorname{NMDAR}(n=10)$ & $\begin{array}{l}\text { Confirmed HSV-1 } \\
\text { cases }(n=52)\end{array}$ & $\begin{array}{l}\text { Confirmed enterovirus } \\
\text { cases }(n=85)\end{array}$ & $\begin{array}{l}\text { Confirmed rabies } \\
\text { cases }(n=6)\end{array}$ \\
\hline \multicolumn{5}{|l|}{ Demographics } \\
\hline Age, median, years & $18.5($ range $11-31)$ & 46.0 (range $0-89$ ) & 14.0 (range $0-75)$ & 38.5 (range $11-72$ ) \\
\hline \multicolumn{5}{|l|}{ Gender } \\
\hline Male & $4(40 \%)$ & $26(50 \%)$ & $52(61 \%)$ & $6(100 \%)$ \\
\hline Female & $6(60 \%)$ & $26(50 \%)$ & $33(39 \%)$ & 0 \\
\hline \multicolumn{5}{|l|}{ Ethnicity } \\
\hline White, Hispanic & $2(20 \%)$ & $8(15 \%)$ & $26(31 \%)$ & $1(17 \%)$ \\
\hline White, non-Hispanic & 0 & $27(52 \%)$ & $30(35 \%)$ & $1(17 \%)$ \\
\hline Black & $3(30 \%)$ & $2(4 \%)$ & $7(8 \%)$ & 0 \\
\hline Asian/Pacific Islander & $5(50 \%)$ & $3(6 \%)$ & $10(12 \%)$ & $2(33 \%)$ \\
\hline Other/unknown & 0 & $12(23 \%)$ & $11(13 \%)$ & $2(33 \%)$ \\
\hline \multicolumn{5}{|l|}{ Clinical findings } \\
\hline \multicolumn{5}{|l|}{ General symptoms } \\
\hline Fever & $7(70 \%)$ & $46(88 \%)$ & $63(74 \%)$ & $4(67 \%)$ \\
\hline GI & $5(50 \%)$ & $19(36 \%)$ & $35(41 \%)$ & $5(83 \%)$ \\
\hline URI & $1(10 \%)$ & $11(21 \%)$ & $24(28 \%)$ & $4(67 \%)$ \\
\hline Rash & 0 & $3(6 \%)$ & $15(18 \%)$ & 0 \\
\hline Intubation & $6(60 \%)$ & $8(15 \%)$ & $4(5 \%)$ & $5(83 \%)$ \\
\hline ICU admission & $8(80 \%)$ & $30(58 \%)$ & $28(33 \%)$ & $4(67 \%)$ \\
\hline Seizures & $8(80 \%)$ & $30(58 \%)$ & $24(28 \%)$ & $2(33 \%)$ \\
\hline Coma & $3(30 \%)$ & $8(15 \%)$ & $5(6 \%)$ & $5(83 \%)$ \\
\hline Died & $1(10 \%)$ & $9(17 \%)$ & $5(6 \%)$ & $6(100 \%)$ \\
\hline \multicolumn{5}{|l|}{ Neurologic symptoms } \\
\hline Altered consciousness & $5(50 \%)$ & $32(62 \%)$ & $31(36 \%)$ & $4(67 \%)$ \\
\hline Ataxia & $6(60 \%)$ & $7(13 \%)$ & $23(27 \%)$ & 0 \\
\hline Focal neurologic signs & $6(60 \%)$ & $17(33 \%)$ & $24(28 \%)$ & $2(33 \%)$ \\
\hline Stiff neck & $3(30 \%)$ & $16(31 \%)$ & $27(32 \%)$ & 0 \\
\hline Cranial nerve abnormality & $3(30 \%)$ & 0 & $3(4 \%)$ & 0 \\
\hline \multicolumn{5}{|l|}{ Psychiatric symptoms } \\
\hline Hallucinations & $6 / 9(67 \%)$ & $8(15 \%)$ & $9(11 \%)$ & $1(17 \%)$ \\
\hline Psychosis & $6 / 9(67 \%)$ & $1(2 \%)$ & $2(2 \%)$ & 0 \\
\hline Personality change & $10(100 \%)$ & $15(29 \%)$ & $7(8 \%)$ & $2(33 \%)$ \\
\hline Irritability & $7(70 \%)$ & $11(21 \%)$ & $29(34 \%)$ & $1(17 \%)$ \\
\hline \multicolumn{5}{|l|}{ Laboratory data $^{\mathrm{a}}$} \\
\hline \multicolumn{5}{|l|}{ CSF } \\
\hline WBC, median (range), per $\mathrm{mm}^{3}$ & 22 (range $3-170$ ) & 90 (range $0-110$ ) & 55.5 (range $0-1,332$ ) & 3.5 (range $0-10)$ \\
\hline Protein, $\mathrm{mg} / \mathrm{dL}$ & 28.5 (range $21-220$ ) & 65 (range 6-297) & 54 (range $16-881)$ & 35 (range 32-172) \\
\hline Glucose, mg/dL & 62 (range $55-72)$ & 68 (range 39-112) & 66 (range 27-159) & 65 (range $60-110$ ) \\
\hline \multicolumn{5}{|l|}{ Neuroimaging abnormality ${ }^{\mathrm{a}}$} \\
\hline \multicolumn{5}{|l|}{ MRI } \\
\hline Temporal lobe & 0 & $36(69 \%)$ & $4(5 \%)$ & 0 \\
\hline Hydrocephalus & 0 & $1(2 \%)$ & 0 & 0 \\
\hline White matter involvement & $1(10 \%)$ & $4(8 \%)$ & $4(5 \%)$ & 0 \\
\hline Cerebral edema & 0 & $1(2 \%)$ & 0 & 0 \\
\hline \multicolumn{5}{|l|}{$\mathrm{CT}$} \\
\hline Temporal lobe & 0 & $14(27 \%)$ & 0 & 0 \\
\hline Hydrocephalus & 0 & $1(2 \%)$ & $3(4 \%)$ & 0 \\
\hline White matter involvement & 0 & $1(2 \%)$ & $2(2 \%)$ & 0 \\
\hline
\end{tabular}


Table 2 (continued)

\begin{tabular}{lllll}
\hline & NMDAR $(n=10)$ & $\begin{array}{l}\text { Confirmed HSV-1 } \\
\text { cases }(n=52)\end{array}$ & $\begin{array}{l}\text { Confirmed enterovirus } \\
\text { cases }(n=85)\end{array}$ & $\begin{array}{l}\text { Confirmed rabies } \\
\text { cases }(n=6)\end{array}$ \\
\hline $\begin{array}{l}\text { Cerebral edema } \\
\text { EEG }\end{array}$ & 0 & 0 & 0 & 0 \\
Slowing & $8(80 \%)$ & $9(17 \%)$ & $7(8 \%)$ & $2(33 \%)$ \\
Epileptiform activity & $1(10 \%)$ & $7(13 \%)$ & $2(2 \%)$ & 0 \\
PLEDs & 0 & $4(8 \%)$ & $1(1 \%)$ & 0 \\
\hline
\end{tabular}

$\mathrm{CSF}=$ cerebrospinal fluid; $\mathrm{WBC}=$ white blood cell; $\mathrm{MRI}=$ magnetic resonance imaging; $\mathrm{CT}=$ computed tomography; $\mathrm{EEG}=$ electroencephalogram; PLEDs = periodic lateralized epileptiform discharges; ICU = intensive care unit

Denominators used in the calculations may vary, depending on the available data

${ }^{a}$ Data are from initial neuroimaging studies or initial lumbar puncture

Despite a good outcome for most patients with antiNMDAR encephalitis, this is a severe disorder that may result in death. In the current study, $10 \%$ of anti-NMDAR+ patients died of this disorder, a number that is higher than those who died of EV (6\%), although lower than the mortality associated with HSV-1 (19\%) and rabies (100\%).

\section{Discussion}

When patients present with clinical features of encephalitis, viral etiologies are among the primary considerations in establishing a diagnosis, with HSV-1 and EV being among the most common offending agents [3, 8,9]. Viral testing can be time consuming and, if it is unable to reveal the cause for the patient's condition, can leave clinicians confounded as to how to proceed with further testing or treatment. Autoimmune etiologies should be considered in the differential diagnosis, even at initial presentation.

Despite the small sample of anti-NMDAR + patients, there are clinical features that appear to be common in patients with anti-NMDAR + encephalitis, and which correlate with those reported in other case series describing similar encephalitic patients. While prodromal symptoms were not highly patterned in the patients in this study, others have noted a consistent 'flu-like' viral illness prior to or with initial presentation, though most patients herein did report fever and headache $[2,10-18]$. The progression of illness noted in this series did, however, resemble that described in other studies.

Anti-NMDAR+ encephalitic patients appear to develop a somewhat predictable course of illness, during which a number of symptoms are frequently observed. Psychiatric symptoms manifest early in the course of illness, and patients are likely to display schizophrenia-like behaviors, such as psychosis and hallucinations. This is consistent with the observation that NMDAR antagonists can cause healthy individuals to develop schizophrenia-like symptoms [19-21]. A newly published study also revealed that antibodies from patients with anti-NMDAR receptor encephalitis cause a dramatic decrease in the number of NMDAR postsynaptic clusters in neuronal cultures, suggesting a decrease in NMDAR function [22]. Behavior and personality changes, sometimes with notable aggression, appear to be additionally remarkable in patients with this form of encephalitis [2, 10-18, 22]. Continued decompensation is the norm in these patients, with most requiring intensive care monitoring and possibly ventilatory support. Hypoventilation, in particular, has been described as a significant feature of this illness, affecting $66 \%$ of those in a 100 -patient series [22]. Other neurologic symptoms also develop in nearly every patient, including dystonias, orofacial movements, particularly oral dyskinesias, and choreoathetosis; none of these have epileptic origins. However, seizures themselves, often of a tonic-clonic nature, are common, with no correlative epileptiform discharges that permitted localization on EEG [2, 16-18, 22]. Mental status changes, though seen in some patients at presentation, consistently developed later in the course of illness, with many patients becoming non-responsive and non-verbal, and displaying low GCS scores. Autonomic instability is almost always observed.

Specific demographic characteristics were notable among our cases and, at times, possibly as a result of the small anti-NMDAR+ sample size, at odds with previous reports. There were a larger number of males in our series, which is likely a result of the selection process. However, it may also be that males have been less readily recognized with this diagnosis, as there have been inconsistent associations with concurrent neoplasms, making clinicians hesitant to attribute symptoms to this specific cause. It is not clear whether or not these individuals would ultimately be found to have tumors, or whether, as with other autoimmune encephalitides (e.g., that are associated with 
voltage-gated potassium channels), a substantial percentage of patients simply did not harbor neoplasms [23, 24]. This is often difficult to ascertain, as many paraneoplastic syndromes can precede the discovery of associated tumor by years [2].

Patients were also more likely to be of Asian or Pacific Islander descent, a finding suggested by a recent publication from Japan [15]. This was surprising, given that teratomas, which are the most common tumors associated with antiNMDAR encephalitis, demonstrate no racial bias, though malignant ones are more common among Asians [25].

In the present series, the median age was younger than that in prior studies, in which a higher percentage of patients with teratomas were identified [2, 7, 10-18, 22]. Given the small number of subjects, it is difficult to ascertain whether the true median age for this entity is as low as 18.5 years, but it is notable that a number of subjects in the current series were children. Those as young as 5 years of age have been described elsewhere, and the median age appears to be distinctly younger than that seen among those with classic paraneoplastic syndromes $[2,14$, 16]. Only $20 \%$ of those examined herein had ovarian teratomas, and, interestingly, of those without tumors, $50 \%$ had positive Mycoplasma IgM serologies. As previously reported, the significance of an isolated positive $M$. pneumoniae serum $\operatorname{IgM}$, especially without signs of Mycoplasma IgG change or polymerase chain reaction (PCR) positivity is unknown [26]. On average, only about $10 \%$ of CEP patients have positive serology for $M$. pneumoniae, making this percentage higher than generally expected. This observation lends itself to the speculation that anti-NMDAR+ patients may develop encephalitis as a result of a post-infectious, antibody-mediated process such as that observed in Sydenham's chorea or other pediatric autoimmune neuropsychiatric disorders associated with streptococcal infections (PANDAs) in which antistreptococcal-antineuronal antibodies are thought to destroy healthy nervous system cells which serve as molecular mimics [27-29]. Because the association with $M$. pneumoniae is not fully understood, clinicians should be cautioned against accepting M. pneumoniae as the sole explanation for an encephalitic illness, especially if this diagnosis is based on a single IgM test.

The differentiation of anti-NMDAR+ associated encephalitis from encephalitis of viral origin on clinical grounds alone is difficult, although our study reveals that there may be some useful clues. HSV-1 patients will typically be older than antiNMDAR+ patients, and are less likely to display severe psychiatric disturbances and schizophrenia-like symptoms. HSV-1 encephalitis patients are also not as likely to display combinations of movement disorders, such as choreoathetosis and orofacial dyskinesias, as those with anti-NMDAR encephalitis [28, 29]. Autonomic instability is not a predominant feature of HSV. Focal EEG abnormalities of the temporal lobe may also point to a viral etiology and, of course, a positive HSV PCR in the CSF is a defining feature of herpes simplex encephalitis [3, 9, 30, 31].

EV encephalitis, like HSV-1 encephalitis, is generally associated with a higher WBC count and protein concentration in the CSF when compared to patients with anti-NMDAR+ encephalitis. Neuroimaging is nonspecific in these patients, but symptomatology can play a substantial role in differentiating these two etiologies because psychiatric and focal neurologic findings will be much less likely. Patients with EV-associated encephalitis are less apt to decompensate and become nonresponsive and unable to verbally communicate. Autonomic instability with subsequent ventilatory support plays a much more subtle role in EV-affected individuals and, as with HSV-1 encephalitis, EV can be detected in the CSF [31,32].

Rabies virus cases pose a particular diagnostic challenge because behavioral symptoms can be as prominent as they are with anti-NMDAR encephalitis. Exposure history, along with diagnostic testing for rabies, is of great value. Typically, more than one diagnostic test will be required to diagnose rabies encephalitis in antemortem patients, while a brainstem biopsy alone is required in post-mortem individuals [33].

The study results do merit a few additional considerations. Reports of cranial nerve abnormalities, ataxic gait, and aphasic findings, obtained from CEP questionnaires, can be subjective in their interpretation. For example, it may be that facial nerve dysfunction and ocular deviations were, in reality, of central origin, as oculogyric crises and facial dystonias have been reported to occur in anti-NMDAR+ patients $[16,22]$. Yet, despite their variable interpretation, gait, facial, and verbal abnormalities, whether of central origin or not, are noted in many cases and can assist in suggesting a diagnosis. Furthermore, though many of our positive confirmed viral cases were not tested for the presence of NMDAR antibodies, samples from 16 patients within the CEP cohort with confirmed viral etiologies were negative for NMDAR antibodies, and based on previous work by Dalmau et al. [22], the antibody is both highly sensitive and specific. It binds to a distinct region of the NMDAR. Although viral encephalitis, stroke, chronic epilepsy, and lupus have been associated with NMDAR antibodies, the region of the NR1 portion of the receptor to which the NMDAR antibodies bind appears to be uniquely associated with anti-NMDAR encephalitis, as opposed to other conditions.

The management of anti-NMDAR+ encephalitis can prove to be very effective and involves the identification and treatment of the tumor, along with immunotherapy. In a series of 100 cases, 91 were women, and $63 \%$ had a tumor, primarily an ovarian teratoma. Only 2 of the 9 men had a tumor (one teratoma of the testis and one small-cell lung cancer). Patients with prompt tumor removal and immunotherapy (e.g., steroids) had better outcomes and fewer 
neurological relapses than those whose tumor was not treated or who had no tumor. Nevertheless, patients of the latter groups also responded to immunotherapy. Overall, $75 \%$ recovered or had mild deficits, while $18 \%$ had severe deficits, with $7 \%$ dying as a result of the encephalitis [22].

Larger studies are needed to estimate the true incidence of anti-NMDAR encephalitis among cases that, after extensive viral screenings, have previously been considered as 'idiopathic encephalitis,' 'encephalitis of unclear etiology,' or 'encephalitis lethargica.' Among these, our study shows that certain clinical profiles such as "encephalitis with dyskinesias or abnormal movements" are likely to be frequently related to anti-NMDAR encephalitis. Of the approximately 20 samples tested, ten $(50 \%)$ were positive, suggesting that this syndrome is a relatively common cause of encephalitis and should be routinely considered in patients with characteristic clinical features. Moreover, although the set of symptoms associated with anti-NMDAR encephalitis is highly predictable and the frequency of dyskinesias very high [34], our study likely underestimates the true incidence of this disorder by focusing on a profile based on 'dyskinesias or abnormal movements,' and excluding milder forms or 'formes frustes,' such as those associated with 'psychiatric symptoms and seizures' with minimal or absent abnormal movements [35].

Anti-NMDAR+ encephalitis is a recognizable, diagnosable, and treatable illness. Patients, in general, will have good outcomes, particularly with aggressive and prompt therapy. Clinicians must be aware of this condition, especially when evaluating patients with encephalitis.

Acknowledgments We thank the laboratory staff of the Viral and Rickettsial Disease Laboratory and Microbial Disease Laboratory for performing the diagnostic testing. We also thank the clinicians who referred cases to the California Encephalitis Project (CEP).

Financial support was obtained from the Centers for Disease Control and Prevention Emerging Infections Program *U50/ CCU915546-09).09 (CG), and there was additional support, in part, from the National Institute of Health 2RO1CA89054-06A2 (JD). There are no conflicts of interest.

Open Access This article is distributed under the terms of the Creative Commons Attribution Noncommercial License which permits any noncommercial use, distribution, and reproduction in any medium, provided the original author(s) and source are credited.

\section{References}

1. Gultekin SH, Rosenfeld MR, Voltz R et al (2000) Paraneoplastic limbic encephalitis: neurological symptoms, immunological findings and tumour association in 50 patients. Brain 123:14811494

2. Tüzün E, Dalmau J (2007) Limbic encephalitis and variants: classification, diagnosis and treatment. Neurologist 13:261-271

3. Glaser CA, Honarmand S, Anderson LJ et al (2006) Beyond viruses: clinical profiles and etiologies associated with encephalitis. Clin Infect Dis 43:1565-1577
4. Seki M, Suzuki S, Iizuka T et al (2008) Neurological response to early removal of ovarian teratoma in anti-NMDAR encephalitis. J Neurol Neurosurg Psychiatry 79(3):324-326

5. Iizuka T, Sakai F (2008) Anti-NMDA receptor encephalitis - clinical manifestations and pathophysiology. Brain Nerve 60:1047-1060

6. Dalmau J, Tüzün E, Wu HY et al (2007) Paraneoplastic anti- $N$ methyl-D aspartate receptor encephalitis associated with ovarian teratoma. Ann Neurol 61:25-36

7. Sansing LH, Tüzün E, Ko MW et al (2007) A patient with encephalitis associated with NMDA receptor antibodies. Nat Clin Pract Neurol 3:291-296

8. Chaudhuri A, Kennedy PG (2002) Diagnosis and treatment of viral encephalitis. Postgrad Med J 78:575-583

9. Whitley RJ, Gnann JW (2002) Viral encephalitis: familiar infections and emerging pathogens. Lancet 359:507-513

10. Vitaliani R, Mason W, Ances B et al (2000) Paraneoplastic encephalitis, psychiatric symptoms, and hypoventilation in ovarian teratoma. Ann Neurol 58:594-604

11. Koide R, Shimizu T, Koike K et al (2007) EFA6A-like antibodies in paraneoplastic encephalitis associated with immature ovarian teratoma: a case report. J Neurooncol 81:71-74

12. Stein-Wexler R, Wootton-Gorges SL, Greco CM et al (2005) Paraneoplastic limbic encephalitis in a teenage girl with an immature ovarian teratoma. Pediatr Radiol 35:694-697

13. Bataller L, Kleopa KA, Wu GF et al (2007) Autoimmune limbic encephalitis in 39 patients: immunophenotypes and outcomes. J Neurol Neurosurg Psychiatry 78:381-385

14. Rosenbaum T, Gärtner J, Körholz D et al (1998) Paraneoplastic limbic encephalitis in two teenage girls. Neuropediatrics 29:159-162

15. Iizuka $T$, Sakai $F$, Ide $T$ et al (2008) Anti-NMDA receptor encephalitis in Japan: long-term outcome without tumor removal. Neurology 70:504-511

16. Yuasa T, Nemoto H, Kimura A (2003) Four cases of acute reversible limbic encephalitis predominantly affecting juvenile female and presenting with psychosis with minimal changes on MRI. Neurol Med 59:45-50

17. Lee ACW, Ou Y, Lee WK et al (2003) Paraneoplastic limbic encephalitis masquerading as chronic behavioural disturbance in an adolescent girl. Acta Paediatr 92:506-509

18. Muni RH, Wennberg R, Mikulis DJ et al (2004) Bilateral horizontal gaze palsy in presumed paraneoplastic brainstem encephalitis associated with a benign ovarian teratoma. J Neuroophthalmol 24:114-118

19. Olney JW, Newcomer JW, Farber NB (1999) NMDA receptor hypofunction model of schizophrenia. J Psychiatr Res 33:523-533

20. Coyle JT, Tsai G, Goff D (2003) Converging evidence of NMDA receptor hypofunction in the pathophysiology of schizophrenia. Ann N Y Acad Sci 1003:318-327

21. Jentsch JD, Roth RH (1999) The neuropsychopharmacology of phencyclidine: from NMDA receptor hypofunction to the dopamine hypothesis of schizophrenia. Neuropsychopharmacology 20:201-225

22. Dalmau J, Gleichman AJ, Hughes EG et al (2008) Anti-NMDAreceptor encephalitis: case series and analysis of the effects of antibodies. Lancet Neurol 7:1091-1098

23. Vincent A, Buckley C, Schott JM et al (2004) Potassium channel antibody-associated encephalopathy: a potentially immunotherapyresponsive form of limbic encephalitis. Brain 127:701-712

24. Thieben MJ, Lennon VA, Boeve BF et al (2004) Potentially reversible autoimmune limbic encephalitis with neuronal potassium channel antibody. Neurology 62:1177-1182

25. Zalel Y, Piura B, Elchalal U et al (1996) Diagnosis and management of malignant germ cell ovarian tumors in young females. Int J Gynaecol Obstet 55:1-10

26. Christie LJ, Honarmand S, Talkington DF et al (2007) Pediatric encephalitis: what is the role of Mycoplasma pneumoniae? Pediatrics 120:305-313 
27. Rose NR (1998) The role of infection in the pathogenesis of autoimmune disease. Semin Immunol 10:5-13

28. Swedo SE (1994) Sydenham's chorea. A model for childhood autoimmune neuropsychiatric disorders. JAMA 272:1788-1791

29. Murphy ML, Pichichero ME (2002) Prospective identification and treatment of children with pediatric autoimmune neuropsychiatric disorder associated with group A streptococcal infection (PANDAS). Arch Pediatr Adolesc Med 156:356-361

30. Redington JJ, Tyler KL (2002) Viral infections of the nervous system, 2002: update on diagnosis and treatment. Arch Neurol $59: 712-718$
31. Hinson VK, Tyor WR (2001) Update on viral encephalitis. Curr Opin Neurol 14:369-374

32. Muir P, van Loon AM (1997) Enterovirus infections of the central nervous system. Intervirology 40:153-66

33. Plotkin SA (2000) Rabies. Clin Infect Dis 30(1):4-12

34. Florance NR, Davis RL, Lam C et al (2009) Anti-NMDA receptor encephalitis in children and adolescents. Ann Neurol (in press). doi:10.1002/ana.21756

35. Niehusmann P, Dalmau J, Rudlowski C et al (2009) Diagnostic value of $N$-methyl-D-aspartate receptor antibodies in women with new-onset epilepsy. Arch Neurol 66:458-464 